\title{
ABELIAN THEOREMS FOR TRANSFORMABLE BOEHMIANS
}

\author{
DENNIS NEMZER \\ Department of Mathematics \\ California State University, Stanislaus \\ Turlock, California 95380 \\ (Received January 27, 1993 and in revised form March 26, 1993)
}

\begin{abstract}
A class of generalized functions called transformable Boehmians contains a proper subspace that can be identified with the class of Laplace transformable distributions. In this note, we establish some Abelian theorems for transformable Boehmians.
\end{abstract}

KEY WORDS AND PHRASES. Abelian theorem, Boehmian, convolution, generalized function, Laplace transform.

1992 MATHEMATICS SUBJECT CLASSIFICATION. Primary 44A10, 44A40. Secondary $46 \mathrm{~F} 99$.

\section{INTRODUCTION AND PRELIMINARIES}

The Laplace transform has recently been extended to a class of generalized functions called transformable Boehmians [1]. The object of this note is to present some Abelian theorems of the initial type for transformable Boehmians. Such theorems relate the behavior of a transformable Boehmian at zero to the behavior of its transform at infinity.

Our notation is the same as that used in [1]. Let $\Omega$ be a subset of the real line $R$. The space of all continuous complex-valued functions on $\Omega$ will be denoted by $C(\Omega)$. Throughout this note it will be assumed that if $\Omega=(a, b)$ then $a<0$ and $b>0$. The space of all functions $f \in C(R)$ such that $f(t)=0$ for $t<0$ will be denoted by $C_{+}(R)$. The support of a continuous function $f$, denoted by supp $f$, is the complement of the largest open set on which $\mathrm{f}$ is zero.

The convolution product of two functions $f, g \in C_{+}(R)$ is given by

$$
(f * g)(t)=\int_{0}^{t} f(t-u) g(u) d u .
$$


A sequence of continuous nonnegative functions $\left\{\delta_{n}\right\}$ will be called a delta sequence if (i) $\int_{0}^{\infty} \delta_{n}(t) d t=1$ for $n=1,2, \ldots$; and (ii) supp $\delta_{n} \subseteq\left[0, \varepsilon_{n}\right], \varepsilon_{n} \rightarrow 0$ as $n \rightarrow \infty\left(\varepsilon_{n}>0\right)$.

A pair of sequences $\left(f_{n}, \delta_{n}\right)$ is called a quotient of sequences if $f_{n} \in C_{+}(R)$ for $n=1,2, \ldots$, $\left\{\delta_{n}\right\}$ is a delta sequence, and $f_{k} * \delta_{m}=f_{m} * \delta_{k}$ for all $k$ and $m$. Two quotients of sequences $\left(f_{n}, \delta_{n}\right)$ and $\left(g_{n}, \varphi_{n}\right)$ are said to be equivalent if $f_{k} * \varphi_{m}=g_{m} * \delta_{k}$ for all $k$ and $m$. A straightforward calculation shows that this is an equivalence relation. The equivalence classes are called Boehmians. The space of all Boehmians will be denoted by $\beta$ and a typical element of $\beta$ will be written as $x=\frac{f_{n}}{\delta_{n}}$.

By defining addition, multiplication, and scalar multiplication, on $\beta$, i.e. $\frac{f_{n}}{\delta_{n}}+\frac{g_{n}}{\varphi_{n}}=\frac{\left(f_{n} * \varphi_{n}+g_{n} * \delta_{n}\right)}{\delta_{n} * \varphi_{n}}, \frac{f_{n}}{\delta_{n}} * \frac{g_{n}}{\varphi_{n}}=\frac{f_{n} * g_{n}}{\delta_{n} * \varphi_{n}}$, and $\alpha\left(\frac{f_{n}}{\delta_{n}}\right)=\frac{\alpha f_{n}}{\delta_{n}}$, where $\alpha$ is a complex number, $\beta$ becomes a convolution algebra.

Since the Boehmian $\frac{\delta_{n}}{\delta_{n}}$ corresponds to the Dirac delta distribution, we denote it by $\delta$. Moreover, the $n^{\text {th }}$ derivative of $\delta$ is given by the formula $D^{n_{\delta}}=\delta^{(n)}=\frac{\delta_{k}^{(n)}}{\delta_{k}}$, where $\left\{\delta_{n}\right\}$ is any infinitely differentiable delta sequence. In general, the $n^{\text {th }}$ derivative of $x \in \beta$ is given by $D^{n} x=x * \delta(n)$.

By the translation operator on $C_{+}(R)$, we mean the operator $\tau_{d}, d$ real, such that $\left(\tau_{d} f(t)=f(t-d)\right.$. The translation operator can be extended to an element $x=\frac{f_{n}}{\delta_{n}} \in \beta$ by $\tau_{d} x=\frac{\tau_{d} f_{n}}{\delta_{n}}$

DEFINITION 1.1. Let $\Omega$ be an open subset of $R$. A Boehmian $x$ is said to be equal to a continuous function $f$ on $\Omega$, denoted by $x=f$ on $\Omega$, if there exists a delta sequence $\left\{\delta_{n}\right\}$ such that $x^{*} \delta_{n} \in C(R)$ for all $n$ and $x^{*} \delta_{n} \rightarrow f$ uniformly on compact subsets of $\Omega$ as $n \rightarrow \infty$.

Two Boehmians $x$ and $y$ are said to be equal on an open set $\Omega$, denoted by $x=y$ on $\Omega$, if $\mathrm{x}-\mathrm{y}=0$ on $\Omega$.

The support of $x \in \beta$, written supp $x$, is the complement of the largest open set on which $x$ is zero. For example, given any delta sequence $\left\{\delta_{n}\right\}$ and $\varepsilon>0, \delta_{n}(t) \rightarrow 0$ uniformly for $|t|>\varepsilon$ as $n \rightarrow \infty$. Thus, supp $D^{n}=\{0\}$ for $n=0,1,2, \ldots$.

For each $x=\frac{f_{n}}{\delta_{n}} \in \beta$, it is not difficult to show that for each $n$

$$
\text { supp } f_{n} \subseteq \operatorname{supp} x+\operatorname{supp} \delta_{n}
$$

For other results concerning Boehmians see [1], [2], and [3]. 


\section{TRANSFORMABLE BOEHMIANS}

A Boehmian $x$ is said to be transformable if there exists a delta sequence $\left\{\delta_{n}\right\}$ and a nonnegative number $\alpha$ such that $x^{*} \delta_{n} \in C_{+}(R)$ for all $n$ and $x^{*} \delta_{n}=O\left(e^{a t}\right)$ as $t \rightarrow \infty$ for all n. The space of all transformable Boehmians will be denoted by $\beta_{L}$.

If $f \in C_{+}(R)$ such that $f(t)=O\left(e^{a t}\right)$ as $t \rightarrow \infty$ for some real number $\alpha$, then the Laplace transform of $f$ is given by

$$
F(s)=\mathcal{L}[f](s)=\int_{0}^{\infty} e^{-s t} f(t) d t \text { for Re } s>\alpha .
$$

Throughout this note s will denote a complex variable, while t or $\sigma$ will denote real variables.

Now, for $x \in \beta_{L}$ where $x^{*} \delta_{n} \in C_{+}(R)$ and $x^{*} \delta_{n}=O\left(e^{\alpha t}\right)$ as $t \rightarrow \infty$ for all $n$, the Laplace Transform $\mathcal{L}[\mathrm{x}](\mathrm{s})=\mathbf{X}(\mathrm{s})$ of $\mathrm{x}$ is defined by the equation $\mathcal{L}[\mathrm{x}](\mathrm{s}) \mathcal{L}\left[\delta_{n}\right](\mathrm{s})=\mathcal{L}\left[\mathrm{x}^{*} \delta_{n}\right](\mathrm{s})$ for all $n$.

It can be shown [1] that the space of transformable distributions $\mathcal{L}_{+}[4]$ is a proper subspace of $\beta_{L}$.

We state without proof the following theorem.

THEOREM 2.1. For $x, y \in \beta_{L}$, if $\boldsymbol{X}(\mathrm{s})$ and $y(s)$ are the Laplace transforms of $x$ and $y$ respectively, then:

1. $\mathcal{L}[x+y](s)=x(s)+y(s)$.

2. $\mathcal{L}[\alpha \mathbf{x}](\mathrm{s})=\alpha \boldsymbol{X}(\mathrm{s}), \alpha$ a complex number.

3. $\mathcal{L}\left[D^{n} x\right](s)=s^{n} x(s)$.

4. $\mathcal{L}\left[x^{*} y\right](s)=x(s) y(s)$.

5. $\mathcal{L}\left[\tau_{d} x\right](s)=e^{-d s} x(s), d$ a real number.

6. If $\boldsymbol{X}(\mathrm{s})=0$, then $\mathrm{x}=0$.

The next theorem will be needed in the proof of an Abelian theorem (Theorem 3.2) in the next section. Also, since the Laplace transform of a Boehmian is an analytic function in some half-plane [1], Theorem 2.2 gives a necessary condition for an analytic function to be the Laplace transform of a Boehmian.

THEOREM 2.2. Let $x \in \beta_{L}$. For each $k$ and $\varepsilon>0, \sigma k e^{-\varepsilon \sigma} X(\sigma)=o(1)$ as $\sigma \rightarrow \infty$.

PROOF. Let $x=\frac{f_{n}}{\delta_{n}} \in \beta_{L}$. We may assume that $f_{n} \in C^{\infty}(R)$ for all $n$. For, if $\left\{\psi_{n}\right\}$ is an infinitely differentiable delta sequence ( that is, $\psi_{n} \in C^{\infty}(R)$ for all $n$ ), then $x=\frac{f_{n} * \psi_{n}}{\delta_{n} * \psi_{n}}$ and $f_{n} * \psi_{n} \in C^{\infty}(R)$ for all $n$.

Assume that supp $\delta_{n} \subseteq\left[0, a_{n}\right]$ for all $n$, where $a_{n} \rightarrow 0$ as $n \rightarrow \infty$. Now, by the Mean Value 
Theorem for Integrals, for each $n$ there exists an $\xi$ (which depends on $n$ and $\sigma$ ) such that $0 \leq \xi \leq a_{n}$ and $\mathcal{L}\left[\delta_{n}\right](\sigma)=\int_{0}^{\infty} e^{-\sigma t} \delta_{n}(t) d t=e^{-\sigma \xi} \int_{0}^{\infty} \delta_{n}(t) d t=e^{-\sigma \xi} \geq e^{-a_{n} \sigma}$

Hence, given an $\varepsilon>0$, we may pick $m$ such that $0<a_{m}<\varepsilon$. Then,

$$
|\boldsymbol{x}(\sigma)|=\frac{\left|\mathcal{L}\left[\mathrm{f}_{\mathrm{m}}\right](\sigma)\right|}{\mathcal{L}\left[\delta_{\mathrm{m}}\right](\sigma)} \leq \mathrm{e}^{\mathrm{a}_{\mathrm{m}} \sigma}\left|\mathcal{L}\left[\mathrm{f}_{\mathrm{m}}\right](\sigma)\right|<\mathrm{e}^{\varepsilon \sigma}\left|\mathcal{L}\left[\mathrm{f}_{\mathrm{m}}\right](\sigma)\right| .
$$

Also, since $f_{m} \in C^{\infty}(R)$, for each nonnegative integer $k$

$$
\sigma^{k}\left[f_{m}\right](\sigma)=O(1) \text { as } \sigma \rightarrow \infty
$$

Hence, (2.1) and (2.2) give

$$
\mathrm{e}^{-\varepsilon \sigma \sigma^{k}} \mathcal{L}[\mathrm{x}](\sigma)=\mathrm{O}(1) \text { as } \sigma \rightarrow \infty \text {. }
$$

The proof is completed by observing that (2.3) is valid for all $\varepsilon>0$ and all nonnegative integers $k$.

THEOREM 2.3. Suppose that $F(s)$ is an analytic function in some half-plane Re $s>a$ and for some integer $k$ and all $\varepsilon>0$ that $s^{k} e^{-\varepsilon s} F(s)=O(1)$ as $s \rightarrow \infty, \operatorname{Re} s>\alpha$. Then, there exists $x \in \beta_{L}$ such that $\mathcal{L}[x](s)=F(s)$, Re $s>\alpha$.

PROOF. Suppose that for some integer $k$ and all $\varepsilon>0$

$$
s^{k} e^{-\varepsilon s} F(s)=O(1) \text { as } s \rightarrow \infty, \operatorname{Re} s>\propto \text {. }
$$

Let $\left\{\delta_{n}\right\}$ be an infinitely differentiable delta sequence. Define $\varphi_{n}(t)=\delta_{n}\left(t-\frac{1}{n}\right)$ for $n=1,2, \ldots$ Thus, $\left\{\varphi_{n}\right\}$ is an infinitely differentiable delta sequence and for each integer $k$ and all $n$

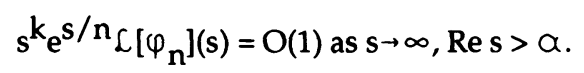

Hence, by (2.4) and (2.5), for each $n$ there exists an $M$ such that $\left|\mathcal{L}\left[\varphi_{n}\right](s) F(s)\right| \leq \frac{M}{|s|^{2}}$ for

Re $s>\alpha$. For $n=1,2, \ldots$, define $f_{n}(t)=\frac{1}{2 \pi i} \int_{Y-i \infty}^{Y+i \infty} e^{s t} \mathcal{L}\left[\varphi_{n}\right](s) F(s) d s$, where $Y>\alpha$.

Then, for each $n, f_{n}$ is a continuous function such that: supp $f_{n} \subseteq[0, \infty), f_{n}(t)=O\left(e^{\gamma t}\right)$ as $\mathrm{t} \rightarrow \infty$, and $\mathcal{L}\left[\mathrm{f}_{\mathrm{n}}\right](\mathrm{s})=\mathcal{L}\left[\varphi_{\mathrm{n}}\right](\mathrm{s}) \mathrm{F}(\mathrm{s})$, Re $\mathrm{s}>\alpha$.

Now, $\mathcal{L}\left[f_{n}{ }^{*} \varphi_{m}\right]=\mathcal{L}\left[f_{n}\right] \mathcal{L}\left[\varphi_{m}\right]=\left(\mathcal{L}\left[\varphi_{n}\right] F\right) \mathcal{L}\left[\varphi_{m}\right]=\left(\mathcal{L}\left[\varphi_{m}\right] F\right) \mathcal{L}\left[\varphi_{n}\right]=\mathcal{L}\left[f_{m}\right] \mathcal{L}\left[\varphi_{n}\right]$

$=\mathcal{L}\left[f_{m}{ }^{*} \varphi_{n}\right]$ for all $n$ and $m$. Thus, $f_{n}{ }^{*} \varphi_{m}=f_{m}{ }^{*} \varphi_{n}$ for all $n$ and $m$. Let $x=\frac{f_{n}}{\varphi_{n}} \in \beta_{L}$. Hence $\mathcal{L}[\mathrm{x}](\mathrm{s})=\mathrm{F}(\mathrm{s})$, $\operatorname{Re} \mathrm{s}>\alpha$.

Although the condition in the previous theorem is sufficient for an analytic function to be the Laplace transform of a Boehmian, as the next example demonstrates it is not necessary .

EXAMPLE 2.4. The transformable Boehmian $x=\sum_{n=0}^{\infty} \frac{\delta^{(n)}}{(2 n) !}$ has Laplace transform $\boldsymbol{X}(\mathrm{s})=\cosh \sqrt{\mathrm{s}}[1]$ 
REMARK 2.5. It is not difficult to show that the transform of a Boehmian is an analytic function in some half-plane [1]. Hence, Theorem 2.2 provides a necessary condition for an analytic function to be the Laplace transform of some transformable Boehmian. Thus, the entire function $g(s)=e^{s}$ is not the transform of a Boehmian. But, for each $\alpha>1$ there is a transformable Boehmian $x_{a}$ such that $x_{\alpha}(s)$ is an entire function and for each $\varepsilon>0, x_{\alpha}(s)=O(\exp |s|(\varepsilon+(1 / \alpha)))$, as $|s| \rightarrow \infty[1]$ (where this relation does not hold for any $\varepsilon<0$ ). An interesting open problem is to characterize the class of transformable Boehmians by their Laplace transforms.

\section{INITIAL VALUE THEOREMS}

In classical analysis there are many different types of Abelian theorems (see [5] and [6]). Abelian theorems of the final type relate the behavior of a function at infinity to the behavior of its transform at zero, while Abelian theorems of the initial type relate the behavior of a function at zero to the behavior of its transform at infinity. It is both interesting and important to extend such theorems to certain classes of generalized functions (see [4], [7], [8], and [9]). For example, Zemanian [4] has extended two Abelian theorems to transformable distributions. In [1] we presented an Abelian theorem of the final type for transformable Boehmians. In this section we will establish three Abelian theorems of the initial type (Theorems 3.2, 3.5, and 3.8).

A real-valued function $m \in C_{+}[a, b]\left(C_{+}[a, b]=C_{+}(R) \cap C[a, b]\right)$ is said to be in $\mathcal{M}$ if $m$ does not change sign in $[a, b]$ and $[\mathcal{L}[\mathrm{m}](\sigma)]^{-1}=\mathrm{O}\left(\sigma^{\mathrm{n}} \mathrm{e}^{\mathrm{o}(\sigma)}\right)$ as $\sigma \rightarrow \infty$ (for some integer $\mathrm{n}$ ).

DEFINITION 3.1. $x, y \in \beta_{L^{*}} x \sim y$ as $t \rightarrow 0^{+}$if there exist $f, g \in C_{+}[a, b], m \in \mathcal{M}$, and an integer $n$ such that $x=D^{n} f$ and $y=D^{n} g$ on some neighborhood of $[a, b], \underset{t \rightarrow 0^{+}}{\operatorname{Lim}} \frac{f(t)}{m(t)}=1$, and $\operatorname{Lim}_{t \rightarrow 0^{+}} \frac{g(t)}{m(t)}=1$

THEOREM 3.2. Suppose $x, y \in \beta_{L}$ such that $x \sim y$ as $t \rightarrow 0^{+}$. Then $\underset{\sigma \rightarrow \infty}{\operatorname{Lim}} \frac{\boldsymbol{x}(\sigma)}{\mathbf{y}(\sigma)}=1$.

PROOF. $x$ can be written in the form

$$
\mathrm{x}=\mathrm{D}^{\mathrm{n}_{\mathrm{f}}+\mathrm{w}} \text {, }
$$

where $w \in \beta_{\mathrm{L}}$ and supp $w \subseteq[\alpha, \infty)(\alpha>0)$. Now, by a classical Abelian theorem (see [5]), $\frac{\mathcal{L}\left[\mathrm{D}^{\mathrm{n}} \mathrm{f}\right](\sigma)}{\sigma^{\mathrm{n}} \mathcal{L}[\mathrm{m}](\sigma)}=\frac{\mathcal{L}[\mathrm{f}](\sigma)}{\mathcal{L}[\mathrm{m}](\sigma)} \rightarrow 1$ as $\sigma \rightarrow \infty$.

Since y can also be written in the form of (3.1), to complete the proof it suffices to show that

$$
\frac{\mathcal{L}[\mathrm{w}](\sigma)}{\sigma^{\mathrm{n}} \mathcal{L}[\mathrm{m}](\sigma)} \rightarrow 0 \text { as } \sigma \rightarrow \infty \text {. }
$$


Now, $w=\frac{f_{n}}{\delta_{n}}$ and by $(1.1)$ supp $f_{n} \subseteq[a, \infty)$ for all $n$. For each $n$, let $g_{n} \in C(R)$ be defined by $\tau_{\alpha} g_{n}=f_{n}$ and let $z=\frac{g_{n}}{\delta_{n}} \in \beta_{L}$. Thus $w=\tau \alpha z$. Then, (for some $\gamma>0$ ),

$$
\frac{\mathcal{L}[w](\sigma)}{\sigma^{n} \mathcal{L}[\mathrm{m}](\sigma)}=\frac{\mathrm{e}^{-\alpha \sigma} \mathcal{L}[\mathrm{z}](\sigma)}{\sigma^{\mathrm{n}} \mathcal{L}[\mathrm{m}](\sigma)}=\mathrm{O}\left(\mathrm{e}^{-\gamma \sigma} \mathcal{L}[\mathrm{z}](\sigma)\right) \text { as } \sigma \rightarrow \infty
$$

By applying Theorem 2.2 to (3.3) we obtain (3.2), and hence the proof is complete.

EXAMPLE 3.3. Let $x \in \beta_{L}$ such that $x=\delta$ on some neighborhood of $[a, b]$. Since $D^{2} t=\delta$, $x \sim \delta$ as $t \rightarrow 0^{+}$and hence $\operatorname{Lim} \boldsymbol{x}(\sigma)=1$.

$$
\sigma \rightarrow \infty
$$

REMARK 3.4. In Definition 3.1 , the condition that the functions $f, g$, and $m$ be continuous may be relaxed. We need only require that $f, g, m \in L^{1}(a, b)$. For if $f \in L^{1}(a, b)$ and $x=D^{n_{f}}$ in some neighborhood of $[a, b]$, then $x=D^{n+1}(l * f)$ in that neighborhood, where $l$ is the Heaviside function $\left(\ell(t)=1\right.$ for $t>0$ and zero otherwise). Also, if $\frac{f(t)}{m(t)} \rightarrow 1$ as $t \rightarrow 0^{+}$, then $\frac{\left(l^{*} \mathrm{f}\right)(\mathrm{t})}{\left(l^{*} \mathrm{~m}\right)(\mathrm{t})} \rightarrow 1$ as $\mathrm{t} \rightarrow 0^{+}$.

THEOREM 3.5. If $x \in \beta_{L}$ such that, for some $f \in L^{1}(a, b), x=D^{n_{f}}$ on some neighborhood of $[a, b]$ and $\frac{f(t)}{t^{\lambda}} \rightarrow \alpha$ as $t \rightarrow 0^{+}(\alpha$ complex and $\lambda$ real, $\lambda>-1)$, then $\lim _{\sigma \rightarrow \infty} \frac{\sigma^{\lambda-n+1} x(\sigma)}{\Gamma(\lambda+1)}=\alpha$ (where $\left.\Gamma(\lambda+1)=\int_{0}^{\infty} \mathrm{e}^{-t_{t} \lambda} d t\right)$.

PROOF. If $\alpha \neq 0$, use Theorem 3.2 with $x=\alpha^{-1} x$ and $y=D^{n_{t}} \lambda$. Suppose $\alpha=0$. Now, $x$ can be written in the form $x=D^{n_{f+w}}$, where $w \in \beta_{L}$ and supp $w \subseteq[\alpha, \infty)(\alpha>0)$. Thus,

$$
\frac{\sigma^{\lambda-n+1} x(\sigma)}{\Gamma(\lambda+1)}=\frac{\sigma^{\lambda+1} F(\sigma)}{\Gamma(\lambda+1)}+\frac{\sigma^{\lambda-n+1} \mathcal{L}[w](\sigma)}{\Gamma(\lambda+1)} \text {. }
$$

By a classical Abelian theorem (see [5]), the first term on the right hand side of (3.4) tends to zero (as $\sigma \rightarrow \infty$ ). By using a similar argument as in the proof of Theorem 3.2, the second term on the right hand side of (3.4) also tends to zero (as $\sigma \rightarrow \infty$ ). This completes the proof.

EXAMPLE 3.6. Let $x=a t^{\lambda}+\sum_{n=0}^{\infty} \frac{\tau_{\varepsilon} \delta^{(n)}}{(2 n) !}$ (where $\varepsilon>0, \lambda>-1$, and $\alpha$ is complex). Since $x=\alpha t^{\lambda}$ on $[0, \varepsilon / 2)$, Theorem 3.5 yields 


$$
\operatorname{Lim}_{\sigma \rightarrow \infty} \frac{\sigma^{\lambda+1} x(\sigma)}{\Gamma(\lambda+1)}=\alpha \quad\left(\sum_{n=0}^{\infty} \frac{\tau_{\varepsilon} \delta^{(n)}}{(2 n) !}=\underset{n}{(-\operatorname{Lim}} \sum_{k=0}^{n} \frac{\tau_{\varepsilon} \delta^{(k)}}{(2 k) !} \text { (see [1]) }\right)
$$

REMARK 3.7. Example 3.6 maybe used to show that Theorem 3.5 cannot be extended to a half-plane. Indeed, let $x=t+\backslash i \backslash s u\left(n=0, \infty, \backslash f\left(\tau_{\pi} \delta^{(n)},(2 n) !\right)\right)$. Then, $x=t$ in $[0,1)$ and $x(s)=\frac{1}{s^{2}}+\sum_{n=0}^{\infty} \frac{\mathcal{L}\left[\tau \pi^{(n)}\right](s)}{(2 n) !}=\frac{1}{s^{2}}+e^{-\pi s} \sum_{n=0}^{\infty} \frac{s^{n}}{(2 n) !}=\frac{1}{s^{2}}+e^{-\pi s} \cosh \sqrt{s}$ (see [1]).

Moreover, for Res $=Y_{0}>0$, there exist positive constants $M$ and a such that $\left|\mathrm{s}^{2} \mathrm{e}^{-\pi \mathrm{s}} \cosh \sqrt{\mathrm{s}}\right| \geq \mathrm{M}|\mathrm{s}|^{2} \mathrm{e}^{\mathrm{a} \sqrt{|s|}} \rightarrow \infty$ as $\mathrm{s} \rightarrow \infty$. Thus, $\mathrm{s}^{2} X(\mathrm{~s})=1+\mathrm{s}^{2} \mathrm{e}^{-\pi s} \cosh \sqrt{\mathrm{s}} \rightarrow \infty$ as $s \rightarrow \infty, \operatorname{Re} s=Y_{0}>0$. Hence, in order to extend Theorem 3.5 (i.e. not restricting $\sigma$ to the real axis) we will require some restrictions.

Let $\mathcal{A}$ denote the set of all Boehmians $x$ such that for each $\varepsilon>0, e^{-\varepsilon s} \boldsymbol{X}(s)=O(1)$ as $s \rightarrow \infty$ for $|\arg s| \leq \psi<\pi / 2$.

THEOREM 3.8. Suppose $x \in \beta_{L}$ such that $x=D^{n_{f}}+\tau_{d} y \quad(d>0, n$ an integer $)$ for some $f \in C_{+}[a, b]$ and $y \in \mathcal{A}$. If $\backslash a\left(, \operatorname{Lim}, t \rightarrow 0^{+}\right) \backslash f\left(f(t), t^{\lambda}\right)=\alpha(\alpha$ complex and $\lambda$ real, $\lambda>-1)$ then $\operatorname{Lim}_{s \rightarrow \infty} \frac{s^{\lambda-n+1} x(s)}{\Gamma(\lambda+1)}=\alpha,|\arg s| \leq \psi<\pi / 2$.

PROOF. $\frac{s^{\lambda-n+1} x(s)}{\Gamma(\lambda+1)}=\frac{s^{\lambda+1} F(s)}{\Gamma(\lambda+1)}+s^{\lambda-n+1} \mathcal{L}\left[\tau_{d} y\right](s)$.

Since $\frac{f(t)}{t^{\lambda} \rightarrow \alpha \text { as } t \rightarrow 0^{+}}$, by a classical Abelian theorem (see [6]), $\frac{s^{\lambda+1} F(s)}{\Gamma(\lambda+1)} \rightarrow a$ as $s \rightarrow \infty$, $\operatorname{larg} s \mid \leq \psi<\pi / 2$. Thus to complete the proof we need only to show that

$$
s^{\lambda-n+1} \mathcal{L}\left[\tau_{d} y\right](s) \rightarrow 0 \text { as } s \rightarrow \infty, \mid \text { arg } s \mid \leq \psi<\pi / 2 \text {. }
$$

Now, for some positive constants $M$ and a

$$
\left|s^{\lambda-n+1} \mathcal{L}\left[\tau_{d} y\right](s)\right| \leq M|\operatorname{Re} s|^{\lambda-n+1} e^{-a R e s},|\arg s| \leq \psi<\pi / 2 .
$$

Since $|\operatorname{Re} s|^{\lambda-n+1} e^{-a \operatorname{Re} s} \rightarrow 0$ as $s \rightarrow \infty$ for $|\arg s| \leq \psi<\pi / 2,(3.5)$ is verified and thus the proof is established.

EXAMPLE 3.9. Let $x$ be as in Example 3.6. Then by Theorem 3.8, $\lim _{s \rightarrow \infty} \frac{s^{\lambda+1} x(s)}{\Gamma(\lambda+1)}=\alpha$, $|\arg s| \leq \psi<\pi / 2$. 
REMARK 3.10. For transformable distributions, Theorem 3.8 may be stated as follows. If $x \in \mathcal{L}_{+}$such that $x=D^{n_{f}}$ in some neighborhood of $[a, b]\left(f \in L^{1}(a, b)\right)$ and $\frac{f(t)}{t^{\lambda}} \rightarrow$ as $t \rightarrow 0^{+}$, then $\lim _{s \rightarrow \infty} \frac{s^{\lambda-n+1} x(s)}{\Gamma(\lambda+1)}=a,|\arg s| \leq \psi<\pi / 2$.

This follows by observing that $x$ can be written as $x=D^{n_{f}}+y$, where $y \in \mathcal{L}_{+}$and supp $\mathrm{y} \subseteq[\xi, \infty)(\xi>0)$. Thus, $y \in \mathcal{A}$ (see [4]).

Since $\mathcal{L}_{+}$is a subspace of $\beta_{L}$, the Abelian theorem of the initial type for $\mathcal{L}_{+}$proved in [4] (Theorem 8.6-2) is a special case of Theorem 3.5. It can be shown [1] that the transformable Boehmian $x$ in Remark 3.7 has Laplace transform $X(s)=\frac{1}{s^{2}}+e^{-\pi s} \cosh \sqrt{s}$, which shows that (i) Theorem 3.5 extends the theorem of Zemanian (since $x$ is not a transformable distribution); and (ii) Theorem 3.8 cannot be extended to a half- plane (see Remark 3.7 ).

ACKNOWLEDGEMENT. The author would like to thank the referee for his/her helpful suggestions and comments.

\section{REFERENCES}

1. Nemzer, D. , The Laplace Transform on a Class of Boehmians, Bull. Austral. Math. Soc. 46 (1992), 347-352.

2. Mikusinski, P. , Convergence of Boehmians, Japan I. Math. 9 (1983), 159-179.

3. Mikusinski, P. , On Harmonic Boehmians, Proc. AMS 106 (1989), 447-449.

4. Zemanian, A.H. , Distribution Theory and Transform Analysis, Dover Publications, New York, 1987.

5. Berg, L. , Operatorenrechnung II: Funktionentheoretische Methoden, Berlin, Dt. Verlag Wiss., 1974.

6. Doetsch, G. , Theorie der Laplace-Transformation. Band I, Verlag Birkhauser, Basel, 1950.

7. Glaeske, H.J. and Müller, D. , Abelsche Satze fur die Laplace-Transformation von Distributionen, Zeitschrift fur Analysis und ihre Anwendungen 10 (1991), 231-238.

8. Lavoine, J. , Théorèmes abéliens et taubériens pour la transformations des distributions, Ann. Soc. Sci. Bruxelles (Ser. I) 89 (1975) , 469-479.

9. Milton, E.O. , Asymptotic Behavior of Transforms of Distributions, Trans. Amer. Math. Soc. 172 (1972), 161-176. 


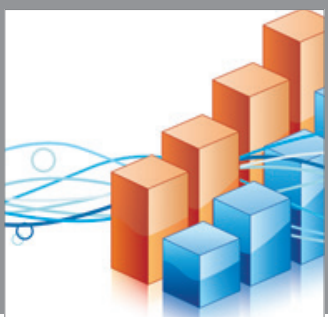

Advances in

Operations Research

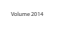

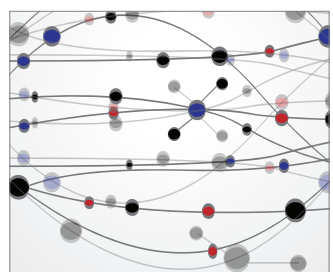

\section{The Scientific} World Journal
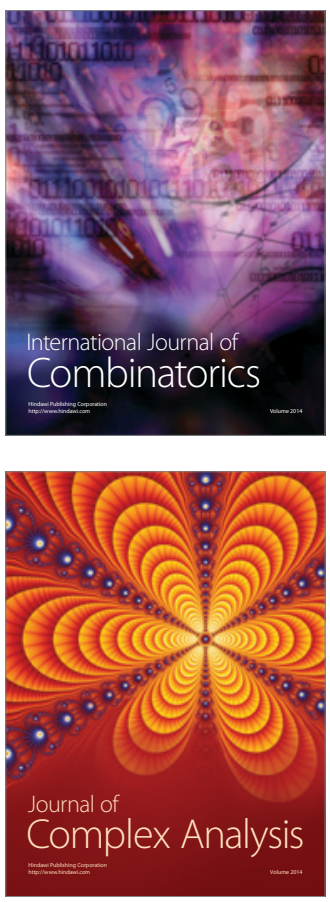

International Journal of

Mathematics and

Mathematical

Sciences
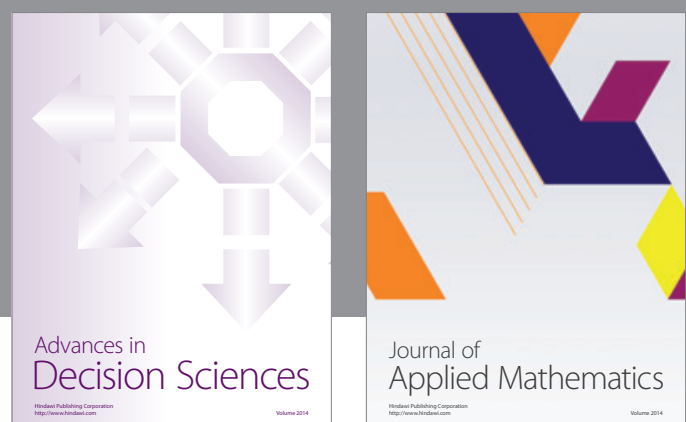

Journal of

Applied Mathematics
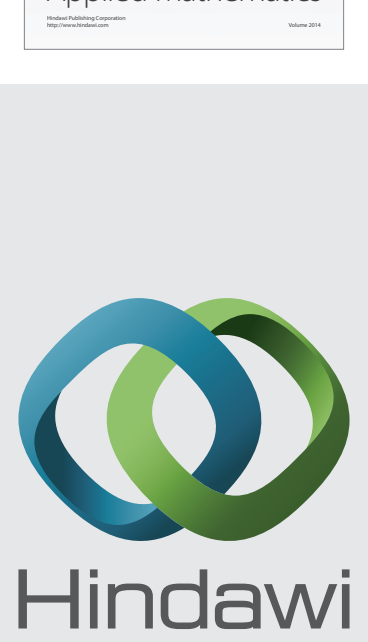

Submit your manuscripts at http://www.hindawi.com
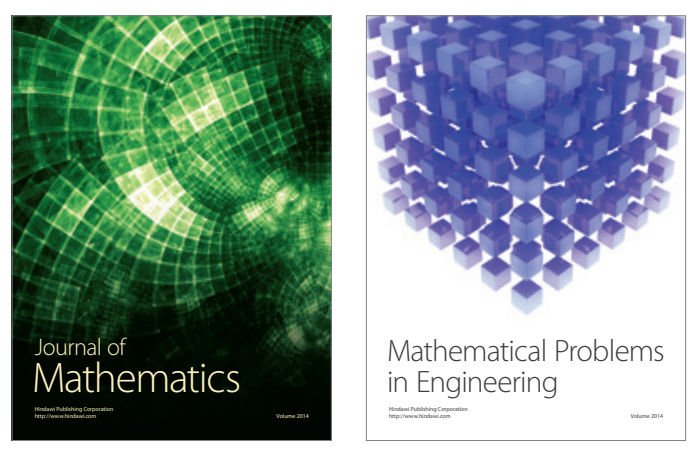

Mathematical Problems in Engineering
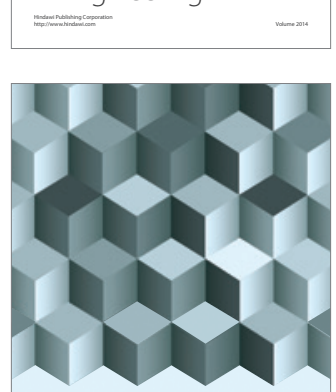

Journal of

Function Spaces
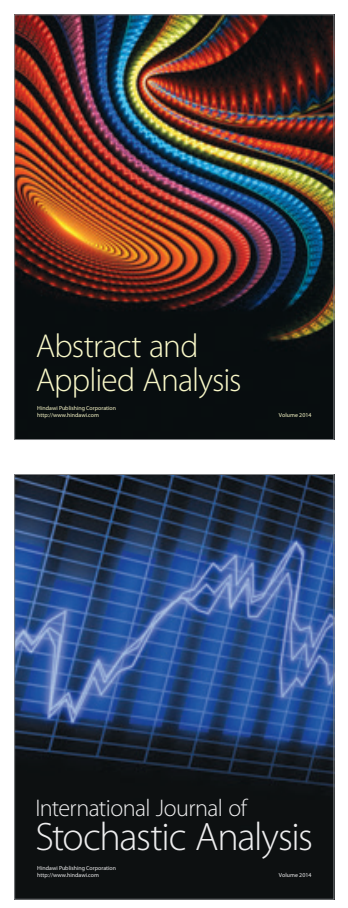

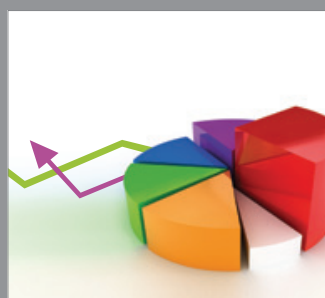

ournal of

Probability and Statistics

Promensencen
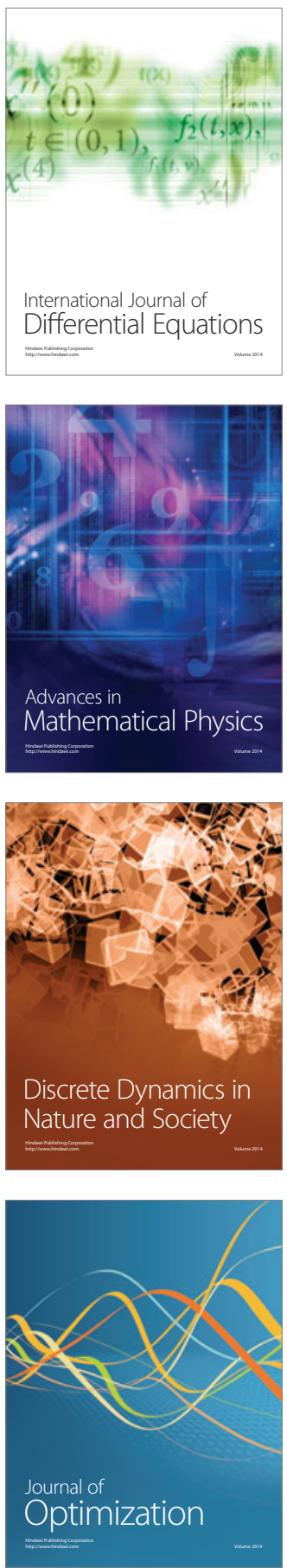